\title{
Mid-IR Imaging of AGB Stars and Circumstellar Modelling
}

\author{
M. Busso, L. Origlia
}

Osservatorio Astronomico di Torino, Pino Torinese, Italy

G. Silvestro, M. Marengo ${ }^{(+)}$

Istituto di Fisica Generale dell'Università, Torino, Italy

${ }^{(+)}$International School for Advanced Studies, Trieste, Italy

P. Persi and M. Ferrari-Toniolo

Istituto di Astrofisica Spaziale del CNR, Frascati, Italy

The evolution of low and intermediate mass $\left(1-8 \mathrm{M}_{\odot}\right)$ stars along the Asymptotic Giant Branch (AGB) is ruled by processes of mass loss, causing the whole convective envelope to be gradually ejected into space. If the stellar mass is sufficiently high $\left(\mathrm{M} \geq 1.5 \mathrm{M}_{\odot}\right)$ the envelope itself becomes enriched in nucleosynthesis products (carbon and $s$-process nuclei) and the star evolves into a C-rich phase. AGB stars are hence surrounded by O-rich or C-rich envelopes, opaque at optical wavelengths, which are best studied through direct imaging in the infrared (IR).
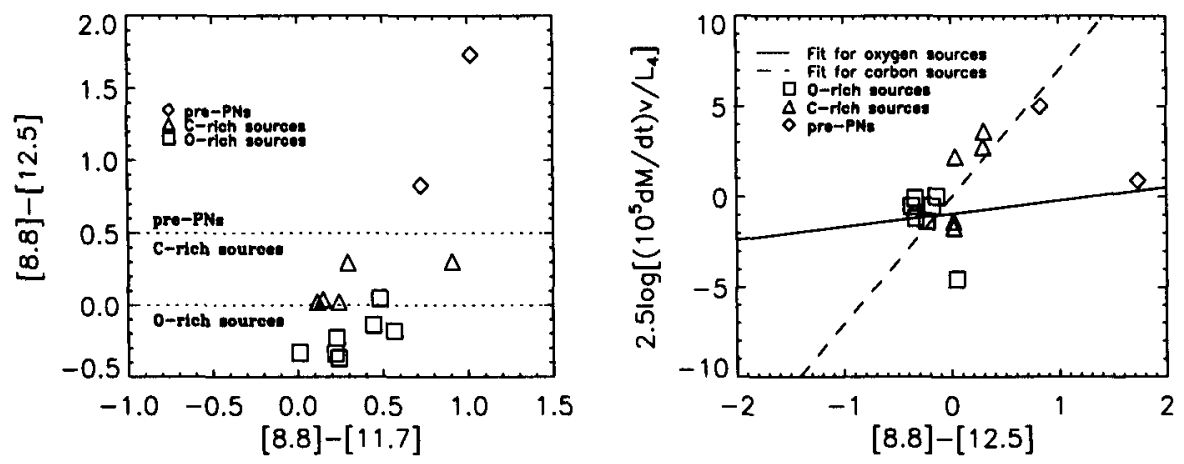

Figure 1. (a) Separation of O-rich AGB stars (squares), C-rich AGB stars (triangles) and pre-PNs (diamonds) by their mid-IR colours. (b) Correlation of the [8.8]-[12.5] colour with mass loss rates.

From the IRAS database we selected 15 bright O-rich and C-rich AGB stars, that we observed with the mid-IR camera Tircam at the TIRGO (Gornergrat, Switzerland) and the S. Pedro Martir (Mexico) IR telescopes. Filters of width $\Delta \lambda=1 \mu \mathrm{m}$ centered at $8.8,9.8,11.7$ and $12.5 \mu \mathrm{m}$ were used. Colour-colour diagrams were then constructed, to search for criteria separating C-rich from O-rich 
sources. Figure 1(a) allows one to see that the [8.8]-[12.5] colour clearly separates the three groups of O-rich AGBs, C-rich AGBs and pre-PNs, in different regions.

In order to investigate the correlations of IR colours with mass loss, we have adopted the mass loss rates estimated by Loup et al. (1993), together with their choice for the values of luminosity, distance and wind velocity. We hence constructed a mass-loss proportional parameter and plotted it against the mid-IR colours. As Figure 1(b) shows, O-rich and C-rich stars both display values of the ([8.8]-[12.5]) colour well correlated with their mass loss rate, but the regression lines are very different. In particular, O-rich stars (probably with a lower mass, $\mathrm{M} \leq 1.3-1.4 M_{\odot}$ ) display a much flatter behaviour. This is an indication that low-mass Miras evolve at lower mass loss rates than more massive red giants, which become C-stars through dredge-up of newly processed carbon. The circumstellar envelopes giving rise to the IR emission were modelled

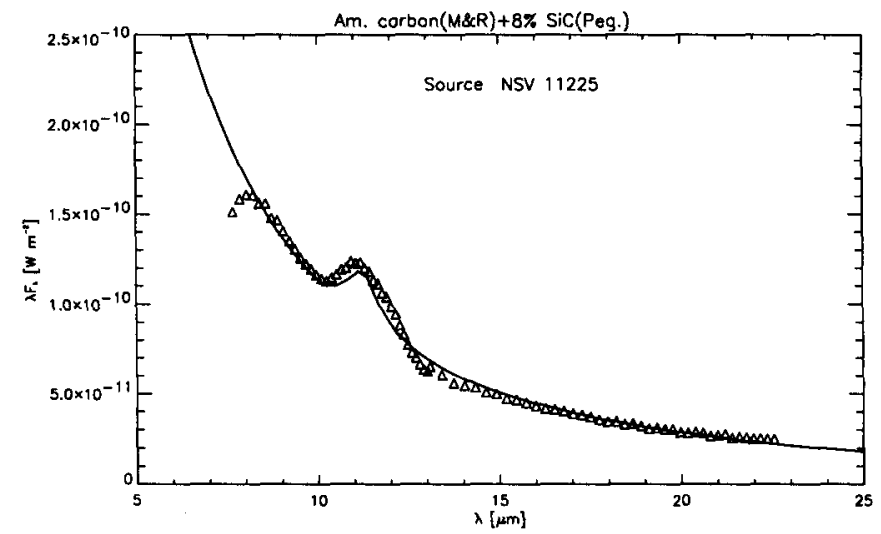

Figure 2. Comparison of the model circumstellar emission with the IRAS LRS spectrum for a C-rich star

through a numerical code, which integrates the equation of non-grey radiative transfer over a spherically symmetric dust shell. The code computes a selfconsistent thermal structure for the envelope, using multiple grain components of carbon- and oxygen-rich material. The spectral energy distribution $\lambda F_{\lambda}$ is calculated taking into account the effect of non-isotropic scattering, absorption and thermal reemission by grains. Model results (Figure 2) can reproduce the IR photometric data of oxygen- and carbon-rich AGB stars with envelopes of different optical depths.

\section{References}

Loup, C., Forveille, T., Omont, A., \& Paul, J., F. 1993, A\&AS, 99, 291 